\title{
A MAC-aware Energy Efficient Reliable Transport Protocol for Wireless Sensor Networks
}

\author{
Sandip Dalvi, Anirudha Sahoo and Ashutosh Deo \\ Department of Computer Science and Engineering \\ Indian Institute of Technology, Bombay, Powai, Mumbai, India 400076 \\ Email: \{sandip_dalvi, sahoo, ashutosh\}@it.iitb.ac.in
}

\begin{abstract}
In a wireless sensor network (WSN), interesting events are reported to the sink by the sensors in a distributed manner. Applications running at the sink require certain reliability in terms of events per unit time to be able to run satisfactorily. Individual report from sensor nodes is not important, but collective reports from sensor nodes of a region of interest of an application are crucial. Thus, the application event rate can be split across different sensor nodes so as to optimize the usage of scarce resources in WSN such as battery power and memory. In this paper, we propose a transport protocol which provides the desired event reliability to the application, by distributing the load at a sensor among its children based on their residual energies and average MAC layer data rate. The event rate distribution happens in such a way that the application at the sink gets its required event rate and the overall energy consumption of nodes is minimized. This protocol can be used for any MAC protocol as long as the average MAC data rate is known. We take the example of two MAC protocols, Slotted CSMA and Probabilistic TDMA. We derive a method for computing average MAC data rate for these two protocols and then show, using simulations, that our transport protocol performs close to optimal.
\end{abstract}

Index Terms- Wireless sensor networks, transport protocol, network lifetime, Slotted CSMA, Probabilistic TDMA

\section{INTRODUCTION}

Wireless Sensor Network (WSN) uses event-driven paradigm to collect data and relies on the collective effort of the sensor nodes in the network. This has several advantages over traditional sensing such as greater accuracy, larger coverage area and extraction of localized features. In order to realize these potential gains, it is imperative that desired events are reliably transported to the sink.

Area monitoring is a typical application of WSNs. In area monitoring, the WSN is deployed over a region where some phenomenon is to be monitored. As an example, a large number of sensor nodes could be deployed over a battlefield to detect enemy intrusion instead of using land mines. When the sensors detect the events being monitored (heat, pressure, sound, light, electro-magnetic field, vibration, etc.), the events need to be reported to the base station or sink node. The data pertaining to such events are required to be transported to the sink reliably. Since data originating from sensor nodes are highly correlated, end-to-end reliability may not be required. However, the applications demand certain event rate at the sink so that it can detect the phenomenon accurately. Hence, unlike the traditional communication networks, WSNs require event-reliability.

The transport protocol described in this paper is devised for delivering such event reliability to the applications, while minimizing the energy consumption of the sensors so that network lifetime is maximized. This protocol is designed for its use in typical WSN applications involving event detection, monitoring and tracking. The sink runs the applications which require certain rates from the sensors in the region of interest. Our protocol has the advantage that it can work with any underlying MAC protocol as long as the MAC layer can specify the average data rate it can provide to the transport layer. Average MAC layer data rate is rate at which a node can send data to its neighboring node taking congestion at the MAC layer into account.

We consider two topologies. The first one is a simple scenario where the sensing node directly communicates with the sink node. We refer to this as single-level load distribution. The second one is a more generic scenario where communication happens in multi-hop from the sensor nodes to the sink node. This is referred to as multi-level load distribution. We present optimization formulation for both the scenarios for a given MAC data rate such that the network lifetime is maximized (network lifetime is defined later in the paper). We then present protocols for the two scenarios and present simulation results which show that our protocols perform very close to the optimal solution. To show how our protocol can work with different MAC protocols, we give examples of two MAC protocols to show how data rate of the two protocols can be computed (taking congestion into account).

\section{RELATED WORK}

In [2], the authors present Event-to-Sink Reliable Transport (ESRT) protocol which provides event-to-sink reliability to the data. It establishes the notion of event-reliability as fidelity of the event stream from source nodes to the sink. The protocol is used in the applications which require that data be fetched continuously from a region of interest. The number of packets reaching the sink in one period, changes as the requested rate changes. The sink then decides at what rate the data should be requested so that the desired event-to-sink reliability is obtained.

The theory of lifetime maximization of unicast sensor networks is given in [8], where an optimal centralized solution is presented in the form of an iterative algorithm. Some of the recent work includes load balancing for data gathering applications [14]. This work tries to solve the problem of energy consuming hot spots in the network by transmitting the packets directly to the sink from the nodes far away from it. It tries to find the balance between the two routing schemes, shortest path routing and direct transmission. The protocol assumes that the sensors have adjustable transmission range, thus enabling them to skip their immediate next hops and transmit directly to the sink. The research reported in [4] aims at selecting the routes and the corresponding power level required to transmit the data to the sink, such that the lifetime of the network is maximized. The routing protocol assumes fixed topology and takes a significant amount of time to arrive at the optimal balance in traffic. The authors in [6] have considered different sensor network deployment strategies to maximize network lifetime by mitigating the problem of energy hogging around the sink. The deployment strategies considered are variable range transmission power control, mobile data sink, multiple data sink and non-uniform initial energy assignment. A distributed joint routing and medium access control algorithm 
is proposed in [10]. In this paper, the authors adopt flow contention graph model for wireless MAC constraints and formulate the maximization of network lifetime as a linear programming problem. The research reported in [13] is also about distributed algorithms to compute an optimal routing scheme to maximize the network lifetime. Cross-layer strategy for lifetime maximization in WSNs is reported in [7], [11]. In [16] the authors present an on-line distributed protocol to solve the problem of energy-minimization with fixed latency constraint for data-gathering in WSNs. An approach to lifetime-maximization through Medium Access control layer is presented in [5], where network lifetime is defined as time till network is functional from the application perspective. In [12], the authors have addressed the problem of scheduling of sensors in a surveillance system such that the lifetime of the system is maximized. They have proposed an optimal solution to find the target-watching schedule for sensors that maximizes system lifetime.

\section{SySTEM MODEL}

We consider a network of sensors with $n$ nodes and $m$ applications. In this paper, we consider routing topology as a tree, i.e., we assume that the routing layer computes a topology to connect the sensing nodes to the sink as a tree. Thus, each node knows its next hop to forward data packets to the sink. In tree topology, each node has only one next hop node on route to the sink. We refer to this next hop node as the parent of that node. A node can be a parent of multiple nodes. We denote the set of children of node $i$ by $S_{i}$. Let $E$ denote the set of applications running in the network and let $N$ denote the set of nodes in the network, excluding the sink node. Our protocol is run in a periodic manner, i.e., the protocol has an observation interval $T$ during which our protocol is run without any change in parameter. In every observation interval, the residual energy is reported by every node to its parent. Then the parent changes rate of reporting of its children for the next observation interval such that the lifetime of the network is maximized.

A node can be associated with an application in two ways: as a sensing node, which generates event of interest or as a routing node, which routes the data towards the sink. In our model, the routing paths from sensing nodes to the sink node form a tree. The leaf nodes of the tree are sensing nodes and the intermediate nodes are routing nodes. We denote the association among applications and nodes by an $n \times m$ matrix $A$, where

$$
A_{i j}= \begin{cases}1 & \text { if } i \text { is a sensing node for application } j \\ 2 & \text { if } i \text { is a routing node for application } j \\ 0 & \text { otherwise }\end{cases}
$$

In any observation period, a sensor node has some amount of energy left, referred to as absolute residual energy. We normalize this with respect to the initial energy and call it as Normalized Residual Energy (NRE). Let $e$ be an $n$ dimensional energy vector, where $e_{i}$ denotes the NRE of node $i$.

When a node transmits or receives a packet, it spends some of its energy. We follow fixed power model, in which a node has to use a fixed amount of power to transmit as well as receive. Thus, it cannot choose its power level based on the distance to the next hop node. Let $E_{t x}$ be the normalized energy required to transmit a packet and $E_{r x}$ be the normalized energy required to receive it. Based upon these values, we define costper-packet for a particular application, as the energy consumed per transmission (for sensing nodes) or forwarding (for routing nodes) of a packet of that application. Let $C$ be an $n \times m$ costper-packet matrix, where each entry $C_{i j}$ corresponds to node $i$ and application $j$, and is given by

$$
C_{i j}= \begin{cases}E_{t x} & \text { if } A_{i j}=1 \\ E_{t x}+E_{r x} & \text { if } A_{i j}=2 \\ 0 & \text { otherwise }\end{cases}
$$

Each application requests data from a region of interest at a certain rate. We define an $m$-dimensional rate vector $d$, whose element $d_{j}$ is the rate at which application $j$ requests data. This rate is nothing but the desired event reliability of that application at the sink. Note that the rates are actually expressed as number of events in an observation period. Also we define an $n$-dimensional MAC rate vector $R^{\text {mac }}(t)$, whose element $R_{i}^{\text {mac }}(t)$ is the average data rate provided by MAC layer of $i^{t h}$ node in the observation period $t$. This rate is nothing but rate at which a node can send data to its parent at MAC layer. We assume that the communication in the entire network happens through one channel which has capacity of transmitting maximum of $B$ events in an observation period.

A node distributes the rate of each application assigned to it by its parent among its children, if it is a routing node for that application. This assigned rate at any given node is called desired event reliability at that node. Let $R_{i j}$ denote the desired event reliability at node $i$ for application $j$. At the start of an observation period $t$, node $i$ gets a new desired reliability value $R_{i j}(t)$ for each application $j$ based on the $R_{i}^{\text {mac }}(t)$ and its NRE. The total rate required by all associated applications on $i^{\text {th }}$ node is given by

$$
R_{i}(t)=\sum_{j \in E} R_{i j}(t)
$$

Let $e_{i d l e}$ be the normalized energy spent per unit time when sensor node is idle. Then the total normalized energy spent in idle period is given by

$$
E_{i}^{\text {idle }}(t)= \begin{cases}T *\left(1-\frac{R_{i}(t)}{B}\right) * e_{\text {idle }} & \text { if } R_{i}(t) \leq B \\ 0 & \text { otherwise }\end{cases}
$$

Energy consumed during an observation interval $t$ by node $i$, or equivalently load on the node $i$ in the interval $t$ is given by

$$
L_{i}(t)=\sum_{j \in E} C_{i j} R_{i j}(t)+E_{i}^{i d l e}(t)
$$

Thus, the NRE of node $i$ at the start of interval $t$ is given by

$$
e_{i}(t)=e_{i}(t-1)-L_{i}(t-1)
$$

Remaining life of node $i$ as estimated at the beginning of interval $t$ is then given by

$$
T_{i}(t)=\frac{e_{i}(t)}{L_{i}(t)}
$$

Note that the remaining life is expressed as the number of observation periods for which the the node will be alive.

We define network lifetime under the load $L_{i}(t)$ as the time elapsed till the first node dies. This definition of network lifetime has been used in [3]. This definition makes the optimization formulation simpler. Furthermore, since the protocols proposed in this paper (presented later), assign reporting rate to the nodes in proportion to their residual energies, typically many more nodes die along with the first node and one or more application becomes defunct. Hence this definition of network lifetime is appropriate for the system proposed in this paper. Thus, network lifetime is given by

$$
\begin{aligned}
T_{N}(t) & =\min _{i \in N} T_{i}(t) \\
& =\min _{i \in N} \frac{e_{i}(t)}{\sum_{j \in E} C_{i j} R_{i j}(t)+E_{i}^{i d l e}(t)}
\end{aligned}
$$

More generally, lifetime of a set of nodes $S$ is defined as the time till the first node in the set $S$ depletes all its energy.

$$
T_{S}(t)=\min _{i \in S} T_{i}(t)
$$

\section{LOAD-DISTRIBUTION STRATEGY}

In this section we present two different load distribution scenarios for our system. The goal of this study is to propose a protocol that can provide the required event reliability while maximizing the network life time. So we present an optimization formulation for the two different load distribution scenarios and then present our protocol for the two scenarios. 
Later we show through simulation that our protocol performs very close to the optimum solution given by the optimization formulation.

\section{A. Single-level Load-distribution}

In this scenario routing tree has only one level, i.e., there is one sink node and there are sensing nodes which are children of the sink node. Note that there is no routing node in this case.

1) Optimization Formulation: Our aim is to have optimum load-distribution in a single level such that network lifetime is maximized. Towards that goal we formulate an optimization problem. Consider a load distribution taking place at the sink node $s . N$ is the set of children of $s$, which are sensing data for various applications running in the network. The optimum solution finds the values of $R_{i j}(t)$ for all $i \in N$ in each observation period $t$, such that the network lifetime is maximized.

$$
\begin{array}{cl}
\max _{R} T_{N}(t) \quad \text { s.t. } \\
\sum_{\substack{i \in N \\
A_{i j} \neq 0}} R_{i j}(t)=d_{j}, \quad \forall j \in E \\
T_{i}(t) \geq T_{N}(t), \quad \forall i \in N \\
R_{i}(t) \leq R_{i}^{\text {mac }}(t), \quad \forall i \in N,
\end{array}
$$

(11) says that the aggregate rate for an application at the children equals the rate at the sink node $s$, which is the total rate of the application. (12) ensures that the estimated life of a child in $N$ is maximized. (13) states rate asked by a parent to its child cannot exceed average data rate provided by MAC of the child node.

Using (5) and (7), an equivalent LP formulation for the above optimization is given as follows.

$$
\begin{gathered}
\min _{R}{ }_{\substack{i \in N \\
A_{i j} \neq 0}} R_{i j}(t)=d_{j}, \quad \forall j \in E \\
\frac{1}{e_{i}(t)}\left(\sum_{j \in E} C_{i j} R_{i j}(t)+E_{i}^{i d l e}(t)\right) \leq \psi, \quad \forall i \in N \\
R_{i}(t) \leq R_{i}^{\text {mac }}(t), \quad \forall i \in N
\end{gathered}
$$

If the above optimal solution is to be implemented, then optimal rates $R_{i j}$ have to be calculated by the sink and communicated to the individual nodes in every observation period. For large network size, the computation overhead could become very high. Hence, we devised a protocol for the singlelevel distribution which has negligible computational overhead compared to the optimal solution and performs very close to the optimal solution.

2) Protocol for single-level load-distribution: We consider a tree topology for single-level distribution. In the beginning of every observation period $t$, node $s$ knows $d_{j}, \forall j \in E$ and every node $i \in N$ knows

1) $R_{i j}(t-1), \forall j \in E$, where $R_{i j}(0)=A_{i j}$

2) $e_{i}(t-1)$

3) $R_{i}^{\text {mac }}(t-1)$

We now present the protocol for single level load distribution. Following steps are taken at the start of observation period $t$ to determine the values of $R_{i j}(t), \forall i, j$.

1) Every node $i \in N$ sends a tuple of $m$ values to the sink node $s$, one corresponding to each application. Each value in the tuple is a fraction of total residual energy of that node which we call as Partial Residual Energy $(P R E)$. Let $P R E_{i j}$ be node $i$ 's partial residual energy corresponding to application $j$. A node also sends its
$R_{i}^{\text {mac }}(t)$ for the observation period. $P R E_{i j}$ is calculated as follows.

$$
\begin{aligned}
P R E_{i j}(t) & =e_{i}(t-1) \times \frac{R_{i j}(t-1)}{L_{i}(t-1)} \\
& =T_{i}(t-1) \times R_{i j}(t-1)
\end{aligned}
$$

We used (7) in the above derivation. We see that $P R E_{i j}$ is the number of packets of application $j$ that node $i$ can send in its remaining lifetime.

2) After receiving this tuple from every child, the node $s$ calculates the distribution of requested rate for each of its child. It computes a share of load for application $j$ given to node $i \in N$ as follows.

$$
R_{i j}(t)=\min \left(d_{j} \times \frac{P R E_{i j}(t)}{\sum_{k \in N} P R E_{k j}(t)}, R_{i}^{\text {mac }}(t)\right)
$$

\section{B. Multi-level Load-distribution}

In this section, we deal with more general tree topology with multiple levels.

1) Optimization Formulation: The optimization aims at finding the values of $R_{i j}(t)$ in observation interval $t$ such that the network lifetime is maximized.

$$
\begin{aligned}
& \max _{R} T_{N}(t) \quad \text { s.t. } \\
& R_{i j}(t)=\sum_{\substack{k \in S_{i} \\
A_{k j} \neq 0}} R_{k j}(t), \quad \forall j \in E, A_{i j}=2 \\
& d_{j}=\sum_{\substack{k \in S_{s} \\
A_{k j} \neq 0}} R_{k j}(t), \quad \forall j \in E, s=\operatorname{sink} \\
& T_{i}(t) \geq T_{N}(t), \quad \forall i \in N \\
& R_{i}(t) \leq R_{i}^{m a c}(t), \quad \forall i \in N
\end{aligned}
$$

(22) and (23) are flow conservation constraints. They make sure that the rate incoming from the children is equal to rate outgoing to its parent. Here $S_{i}$ represents the set of children of node $i$.

An equivalent LP formulation for the same optimization is given as follows.

$$
\begin{gathered}
\min _{R} \text { s.t. } \\
R_{i j}(t)=\sum_{\substack{k \in S_{i} \\
A_{j k} \neq 0}} R_{k j}(t), \quad \forall j \in E, A_{i j}=2 \\
d_{j}=\sum_{\substack{k \in S_{s} \\
A_{j k} \neq 0}} R_{k j}(t), \quad \forall j \in E, s=\operatorname{sink} \\
\frac{1}{e_{i}(t)}\left(\sum_{j \in E} C_{i j} R_{i j}(t)+E_{i}^{i d l e}\right) \leq \psi, \quad \forall i \in N \\
R_{i}(t) \leq R_{i}^{\text {mac }}(t), \quad \forall i \in N
\end{gathered}
$$

The load-distribution strategy used in single-level case balances the load at a parent node among its children based only upon their residual energies. But, to maximize the network lifetime, the load at the parent node should be distributed appropriately among all the nodes contained in its subtree. To achieve this goal, instead of considering remaining lifetime of a node as a basis of distribution for an application (as was done in case of single-level load-distribution), our multi-level protocol considers the application lifetime in the subtree rooted at that node. Application lifetime in a subtree rooted at a node is the time till the first node from the set of nodes in the subtree associated with that application dies. Let us denote the set of nodes in the subtree rooted at node $i$, which are associated with application $j$, as $S_{i j}$. Thus, the lifetime of application $j$ in the subtree rooted at node $i$ is $T_{S_{i j}}$.

Figure 1 shows a network with a tree topology. Singlelevel distribution taking place at node $i$ would balance the 


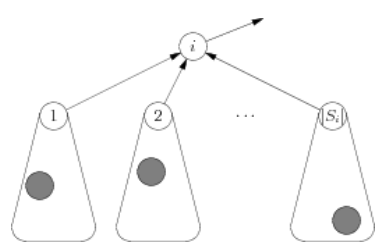

Fig. 1. Topology for Multi-level Distribution

load among the nodes in the set $S_{i}$ with respect to their residual energy levels, which does not consider other nodes in the subtrees rooted at the children of node $i$ (shaded in grey). Some of these nodes may have smaller remaining life than the children themselves. Thus, asking these nodes to gather data at higher rate may deplete their energy faster and hence reduce the network lifetime. Hence, in a multi-level distribution scenario, the load on the grey or weak nodes has to be balanced in proportion to their residual energies. Thus, we design our multi-level protocol as follows.

2) Protocol for Multi-level Load-distribution: In the beginning of every observation period $t$, node $i$ knows (i)

1) $R_{i j}(t-1), \forall j \in E$, where

$$
R_{i j}(0)= \begin{cases}\epsilon & \text { if } A_{i j}=2 \\ A_{i j} & \text { otherwise }\end{cases}
$$

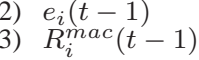

where $\epsilon$ is a tunable parameter of the protocol. A small value of $\epsilon$ would take more time for the protocol to converge to optimal solution, but it will be very close to the optimal solution. On the other hand, a larger value of $\epsilon$ would make the protocol converge fast, but its deviation from optimal solution will be larger.

We now present the protocol for load-distribution in multilevel scenario. Following steps are taken at the start of an observation period $t$.

1) Node $i$ calculates $T_{S_{i j}}(t-1)$ as follows.

$$
T_{S_{i j}}(t-1)=\left\{\begin{array}{l}
\min \left\{T_{S_{k j}}(t-1), T_{i}(t-1)\right\} \forall k \in S_{i j} \\
\text { if } A_{i j} \neq 0 \\
0, \text { otherwise }
\end{array}\right.
$$

2) Node $i$ calculates the values of $P R E_{i j}(t)$ in this step for all $j \in E$ and sends them to its parent. $P R E_{i j}(t)$ is calculated as follows.

$$
P R E_{i j}(t)=T_{S_{i j}}(t-1) \times R_{i j}(t-1)
$$

Thus $P R E_{i j}(t)$ is the number of packets node $i$ can send to its parent before any node in its subtree associated with application $j$ dies. After this step, node $i$ receives the rate $R_{i j}(t)$ for each application for the current period from its parent. In the next step, it distributes this rate among its children.

3) Node $i$ distributes the aggregate load among its children in the similar way as in (20). Thus, rate for application $j$ assigned to node $k \in S_{i}$ is

$$
R_{k j}(t)=\min \left(R_{i j}(t) \times \frac{P R E_{k j}(t)}{\sum_{l \in S_{i}} P R E_{l j}(t)}, R_{k}^{m a c}(t)\right)
$$

At the end of Step 1, node $i$ knows the remaining lifetime of its subtree. This step makes sure that the minimum remaining lifetime of the node in the subtree rooted at $i$ is used to compute $P R E_{i j}$. This ensures that nodes with lower residual energy are asked lesser rate than those with higher residual energy.

\section{ANALYSIS OF MAC LAYERS}

The transport protocol proposed above is independent of the underlying MAC protocol in the sense that the protocol will work with any MAC as long as the average data rate provide by the underlying MAC is known. For a given MAC, this may require modeling and analyzing the MAC during congestion scenario. To illustrate this aspect of our protocol we show the analysis of two MAC protocols which have been studied for WSNs [15]: Slotted CSMA and Probabilistic TDMA to compute average MAC data rate.

\section{A. Slotted CSMA}

Slotted CSMA [15] protocol is similar to regular CSMA but the time is divided into slots. Each transmission is initiated at the start of a slot. When a node has packet to transmit, it chooses an initial random backoff from a given contention window: At the end of backoff count down, it transmits the packet if the channel is idle, otherwise contention window is doubled and a new random backoff is chosen from this new contention window. Let us denote initial contention window size as $C W_{\text {min }}$. So, for each unsuccessful transmission attempt, contention window is doubled until it reaches a maximum $C W_{\max }$ or the maximum retransmission limit $M$ is reached. So, $C W_{\text {max }}=2^{l} * C W_{\text {min }}$, where $l$ is the number of backoff stages. We denote the probability that a packet transmission results in a collision by $p$. Then, in the absence of retransmission limits, the probability that $C W=W$ is given in [9] as

$$
\operatorname{Pr}\{C W=W\}= \begin{cases}p^{k-1}(1-p) & \text { for } W=2^{k-1} C W_{\text {min }} \\ p^{l} & \text { for } W=C W_{\text {max }}\end{cases}
$$

where $k<l$. Note that when the retransmission limit $M<l$, the contention window does not grow to $C W_{\max }$.

We present the following derivation of average backoff window size which is taken from [9]. With probability $1-p$, first transmission is successful and the average backoff window of such packet is $C W_{\min } / 2$. With probability $p(1-p)$, the first transmission fails and the packet is successfully transmitted in the second attempt (using a backoff window of $2 C W_{\text {min }}$ ), which adds $C W_{\min }$ to the average backoff window seen by the packet. Continuing like this for cases with larger numbers of collisions, the average backoff window in the saturated case is given by [9]

$$
\bar{W}= \begin{cases}\frac{1-p-p(2 p)^{l}}{1-2 p} \frac{C W_{\min }}{2} & \text { for } M \geq l \\ \frac{1-p-p(2 p)^{M-1}}{1-2 p} \frac{C W_{\min }}{2} & \text { for } M<l\end{cases}
$$

Assuming that each node has a constant probability of transmission in each idle slot, the probability that a node transmits in a slot is given by $\tau=1 / \bar{W}$. Then the probability $p_{s}$ that a node's transmission is successful given by

$$
p_{s}=1-p=(1-\tau)^{N-1}
$$

The equation (37) can be solved for $p_{s}$. Let $T_{d}$ be the time to transmit payload part of the packet and $T_{p}$ be the time to transmit the complete packet. So the MAC layer utilization is given by

$$
U=\frac{T_{d} * p_{s}}{T_{p} * p+T_{p} * p_{s}}=\frac{T_{d}}{T_{p}} * p_{s}
$$

Let $D^{m a c}$ be the data rate provided by physical layer to the MAC layer, then the data rate of MAC layer, $R^{\text {mac }}$, is given by

$$
R^{m a c}=U * D^{m a c}
$$

This MAC data rate can be used by the transport layer to compute the rate to be assigned to the nodes.

\section{B. Probabilistic TDMA}

The paper presented in [15] gives the following probabilistic model for calculation of utilization for Probabilistic TDMA. We used those equations to derive the average MAC layer data rate of Probabilistic TDMA (P-TDMA). P-TDMA is a MAC protocol, which combines the features of TDMA and CSMA 
into one protocol. Every node in the network is assigned a time slot. But a node can transmit in other node's slot if the slot is found idle. Thus, P-TDMA tends to have more throughput than regular TDMA. Consider a network having $N$ nodes where each node is assigned a unique TDMA time slot and this slot repeats itself after every $N$ slots. Each node transmits in its own slot with probability $a$, and transmits in the other $N-1$ slots with probability $b$.

Consider a TDMA time slot $i$ whose owner is node $O_{i}$. Then, $O_{i}$ could be active (having packet) or inactive (having no packet). Let $N_{c}$ be the number of active nodes at the time of slot $i$. If $O_{i}$ is an active node, then it will transmit in slot $i$ with probability $a$, while the remaining $N_{c}-1$ active nodes will transmit in slot $i$ with probability $b$. Thus, $P_{s}^{a}$, the probability that a packet transmission is successful in a slot owned by an active node is given by [15]

$P_{s}^{a}=a(1-b)^{N_{c}-1}+(1-a) b(1-b)^{N_{c}-2}\left(N_{c}-1\right)$

Now if $O_{i}$ is inactive node, all $N_{c}$ active nodes will transmit in slot $i$ with probability $b$. Hence, $P_{s}^{i n}$, the probability of successful packet transmission in a slot owned by an inactive node is given by [15]:

$$
P_{s}^{i n}=b(1-b)^{N_{c}-1} N_{c}
$$

Let $T_{d}$ be the time to transmit just the payload part of a packet and $T_{p}$ be the time to transmit the entire packet. Then the MAC layer utilization is given by:

$$
U=\frac{P_{s}^{a} * N_{c}+P_{s}^{i n} *\left(N-N_{c}\right)}{N} * \frac{T_{d}}{T_{p}}
$$

Similar to the slotted CSMA case, the data rate of MAC layer is given by

$$
R^{m a c}=U * D^{m a c}
$$

\section{Simulation EXPERIMENTS}

We have simulated the single-level and multi-level distribution protocols described in this paper. The simulator was developed in Java. We used lp_solve 5.1.1.3 package [1] to solve the LP optimization problem presented in this paper to obtain the optimal solution. We analyzed the performance of our protocol in terms of network lifetime and compared it with the optimal solution given by the LP optimization formulation.

We have simulated two scenarios with respect to the initial energy of the sensor nodes.

- Full initial energy: All the nodes start with full energy, i.e., all nodes have normalized energy of one unit. This scenario represents the case when all the sensor nodes are identical to each other.

- Random initial energy: Initial energy of each node is uniformly distributed between 0.25 and 1 unit. This scenario represents the case when the sensor nodes are of different capacity.

Rates for all the applications were also selected randomly between 0 and 100 per observation period with uniform distribution. Values for both $E_{t x}$ and $E_{r x}$ were taken as $10^{-4}$ normalized energy units. $e_{i d l e}$ was set to $10^{-6}$ normalized energy units. Capacity of the channel $(B)$ was set to 160 events per observation interval. Each event was of 120 bytes and an observation period was $100 \mathrm{msec}$. Association of between applications and nodes were done randomly.

We evaluated our protocol in a small and large network to verify that the protocol performs equally well when the network size increases. Small network was represented by 20 nodes in single level case and 50 nodes in multi-level case, whereas large network was represented by 50 nodes in single level case and 80 nodes in multi-level case. However, due to space limitation, we are not able to present the results for large network size.

\section{A. Validation of MAC Models}

We ran simple experiments to validate the probabilistic models developed in Section V. We ran packet level simulation of slotted CSMA and P-TDMA and obtained the MAC data rate in saturation condition. We compared them with the MAC data rate computed as per the probabilistic model given in Section V. Figure 2 compares the MAC data rate (in events per observation period) obtained from simulation and from the model for slotted CSMA MAC for a node. It is clear that the model very closely follows the simulation results. For all the nodes in the network we verified that the model based value is quite close to the simulation based result.

Similarly, Figure 3 shows comparison between simulation based and model based MAC rate for a node running $\mathrm{P}$ TDMA. Here also the model gives results very close to that of simulation.

\section{B. Simulation of Single-level Distribution Protocol}

Figure 4 shows the performance of a single-level distribution with different types of MAC layers with 20 nodes and 10 applications when all the nodes start with full energies. The performance parameter being compared is the network lifetime against observation period. In addition to slotted CSMA and P-TDMA MAC, we have also used a theoretical infinite MAC, which can provide any rate asked by the transport layer. Basically, infinite MAC provides a theoretical upper bound for any practical MAC. In terms of optimization formulation, infinite MAC would mean that there is no MAC rate constraint, i.e., constraint (13) can be taken off from the optimization formulation. We compared performance of each MAC layer with the corresponding optimal solution. For example, for P-TDMA MAC, we ran simulation to plot network lifetime versus observation interval. We also used lpsolve to find the optimal solution of LP formulation presented in Section IV. It can be observed from the figure that the single-level distribution protocol performance is almost same as the optimal solution for all the three different MAC protocols. Also, in all the three cases, the protocol converges instantaneously to the optimal solution. Network life time for infinite MAC is always the highest followed by P-TDMA which is followed by slotted CSMA.

Figure 5 shows the performance of the protocol running on top of different MAC layers on the same network as in Figure 4 with the same application-node associations, but the nodes start out with random initial energies. Here also the performance of the protocol follows the optimal solution very closely for all MAC types and the convergence is also instantaneous. But compared to the full initial energy case, the network lifetime is much smaller, for all the three MACs, because in this case, some nodes start with lower than full energy.

We ran the same experiments with a larger network, i.e., with 10 applications and 50 nodes. The results were similar: the protocol performance was close to the optimal solution. We are not able to provide these results due to space constraint.

In the next experiment, we wanted to verify that the application running at the sink is actually getting the required rate all the time. Figure 6 plots event rate (in events per observation period) at different time (in terms of observation periods). From the figure it is evident that the observed event rate is same as the required event rate of the application most of the time. Thus, this validates that the transport protocol proposed in this paper is in fact able to provide required event rate to the applications. 


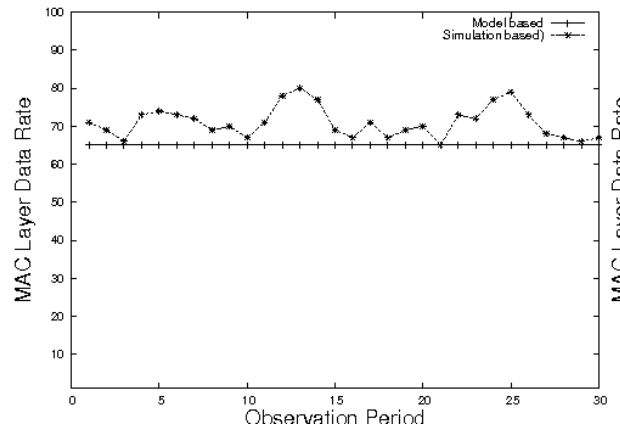

Fig. 2. Slotted CSMA

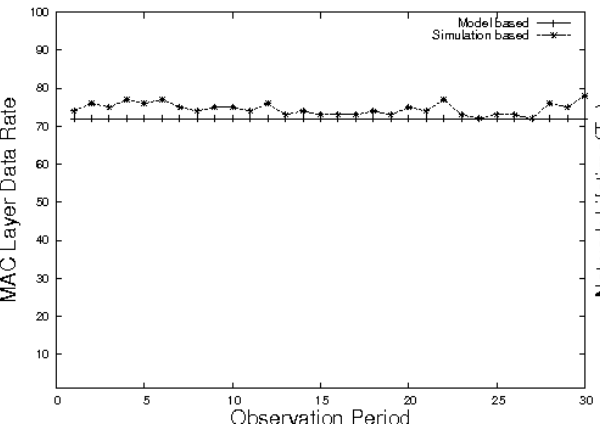

Fig. 3. Probabilistic TDMA

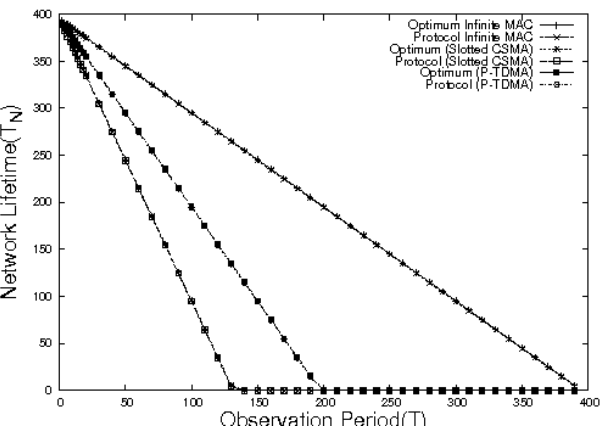

Fig. 4. Single-level Distribution: 10 Applications, 20 Nodes with Full Initial Energy

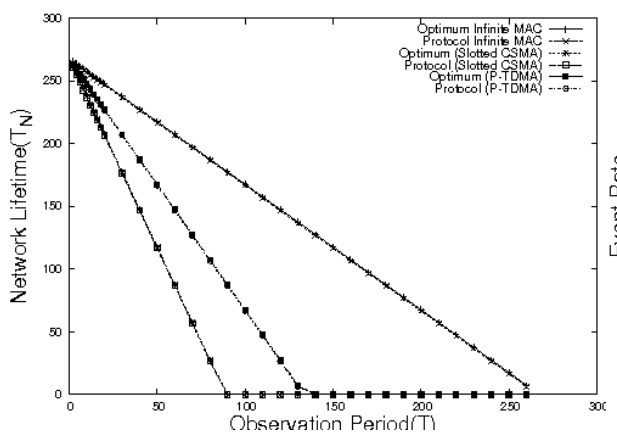

Fig. 5. Single-level Distribution: 10 20 Nodes with Random Initial Energy

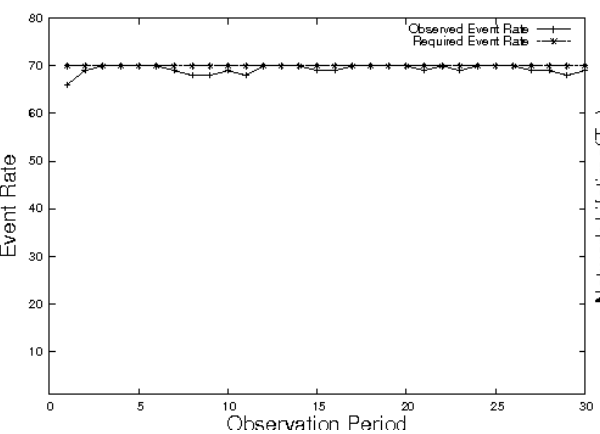

${ }^{10}$ Ohservation Perind

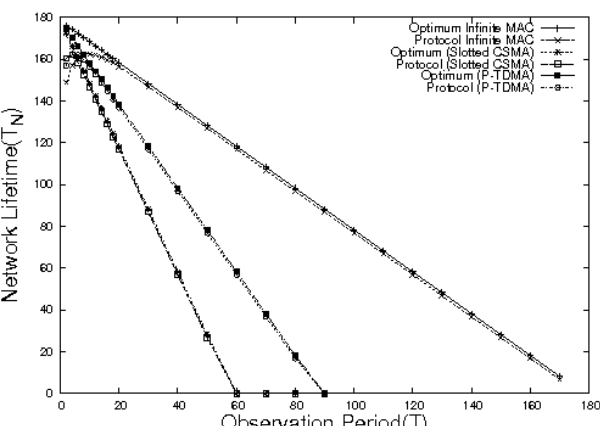

Fig. 6. Comparison of Observed eve
Required Event Rate for an Application

Fig. 7. Multi-level Distribution: 10 Applications, 50 Nodes with Full Initial Energy

\section{Simulation of Multi-level Distribution Protocol}

Figure 7 shows the performance of multi-level distribution with the three different MAC protocols with 50 nodes and 10 applications when all the nodes start with full energies. As can be seen from the figure, the multi-level distribution protocol performs almost exactly same as the optimal solution for all three MAC types. The protocol convergence time (to optimal solution) is little more than the single level case.

We reran the same experiment with a larger network, i.e., with 80 nodes and 10 applications. Here also we observed that the protocol closely followed the optimal solution for all the MAC types. However, we are not able to provide the results due to space limitation.

\section{CONCLUSION}

We presented a protocol which maximizes the lifetime of WSN. The protocol has two versions: one for single-level topology and the other for multi-level topology. We formulated optimization problem for both the topologies. We presented our simulation results to show that both the versions of the protocol perform quite close to the optimal solution. The protocol presented is agnostic to the MAC layer protocol as long as the MAC layer can provide the data rate that it can provide to the transport layer. To illustrate this aspect of our protocol we also presented the model for two MAC protocols: Slotted CSMA and Probabilistic TDMA. We presented simulation results to validate the models and also to show that the observed rate is same as the required rate most of the time. Hence, our protocol can be used to provide reliable event transport in a WSN such that network lifetime is maximized.

\section{REFERENCES}

[1] lpsolve. http://lpsolve.sourceforge.net/5.1/

[2] O.B. Akan and I.F. Akyildiz. Event-to-sink reliable transport in wireless sensor networks. IEEE/ACM Transactions on Networking, 13(5):10031016, October 2005.
[3] J.-H. Chang and L. Tassiulas. Routing for maximum system lifetime in wireless ad hoc networks. In 37th Annu. Allerton Conf. Communication, Control, and Computing, September, 1999.

[4] Jae-Hwan Chang and L. Tassiulas. Energy conserving routing in wireless ad-hoc networks. IEEE INFOCOM, 2000.

[5] Yunxia Chen and Qing Zhao. On the lifetime of wireless sensor networks. IEEE Communications Letters, 9(11):976-978, Novemeber, 2005.

[6] Zhao Cheng, Mark Perillo, and Wendi B. Heinzelman. General network lifetime and cost models for evaluating sensor network deployment strategies. IEEE Transactions on Mobile Computing, 7(4):484-497, April 2008.

[7] Shuguang Cui, S. Lall, and N.A. Goldsmith. Cross-layer design for lifetime maximization in interference-limited wireless sensor networks. IEEE Transactions on Wireless Communications, 5(11):3142-3152, IEEE Transactions

[8] Joseph C. Dagher, Michael W. Marcellin, and Mark A. Neifeld. A theory for maximizing the lifetime of sensor networks. IEEE Transactions on Communications, 52(2):323-332, February, 2007.

[9] Liang Cheng Jun Peng and Biplab Sikdar. A wireless mac protocol with collision detection. In IEEE Transactions on Mobile Computing, VOL. 6, NO. 12, 2007.

[10] Seung-Jun Kim, Xiaodong Wang, and Mohammad Madihian. Distributed joint routing and medium access control for lifetime maximization of wireless sensor networks. IEEE Transactions on Mobile Computing, 6(7):2669-2677, July 2007.

[11] Hojoong Kwon, Tae Hyun Kim, Sunghyun Choi, and Byeong Gi Lee. A cross-layer strategy for energy-efficient reliable delivery in wireless sensor networks. IEEE Transactions on Wireless Communications, 5(12):3689-3699, December, 2006.

[12] Hai Liu, Xiaohua Jia, Peng-Jun Wan, Chih-Wei Yi, S. Kami Makki, and Niki Pissinou. Maximizing lifetime of sensor surveillance systems. IEEE Transactions on Networking, 15(2):334-345, April 2007.

[13] R. Madan and S. Lall. Distributed algorithms for maximum lifetime routing in wireless sensor networks. IEEE Transactions on Wireless Communications, 5:2185-2193, August, 2006.

[14] D. Mandala, Fei Dai, and Xiaojiang Du and Chao You. Load balance and energy efficient data gathering in wireless sensor networks. IEEE International Conference on Mobile Adhoc and Sensor Systems (MASS), 2006.

[15] Ajit Warrier and Injong Rhee. Stochastic analysis of wireless sensor network mac protocols. In Technical report, Computer Science Department, North Carolina State University, Raleigh, NC, 2005.

[16] Yang Yu, V.K. Prasanna, and B. Krishnamachari. Energy minimization for real-time data gathering in wireless sensor networks. IEEE Transactions on Wireless Communications, 5(11):3087-3096, November, 2006. 